\title{
Dual trigger with gonadotropin releasing hormone agonist and human chorionic gonadotropin significantly improves live birth rate for women with diminished ovarian reserve
}

Ming-Huei Lin ${ }^{1,2,3,4+}$, Frank Shao-Ying Wu ${ }^{5,6+}$, Yuh-Ming Hwu ${ }^{1,2,3,4^{*}}$, Robert Kuo-Kuang Lee ${ }^{1,2,7}$, Ryh-Sheng Li ${ }^{1}$ and Sheng-Hsiang $\mathrm{Li}^{2,3}$

\begin{abstract}
Background: Diminished ovarian reserve (DOR) remains one of the greatest obstacles affecting the chance of a successful live birth after fertility treatment. The present study was set to investigate whether using a "dual trigger" consisted of human chorionic gonadotropin ( $\mathrm{hCG}$ ) plus gonadotropin releasing hormone agonist (GnRH-a) for final oocyte maturation could improve the IVF cycle outcomes for patients with diminished ovarian reserve.

Methods: A total of 427 completed GnRH-antagonist downregulated IVF cycles with fresh embryo transfer (ET) were included in this retrospective analysis. DOR was defined as antral follicle count $\leq 5$ and serum anti-Müllerian hormone level $\leq 1.1 \mathrm{ng} / \mathrm{mL}$. The control group $(n=130)$ used a $6500 \mathrm{IU}$ of recombinant hCG for trigger, and the study group $(n=297)$ used $0.2 \mathrm{mg}$ of triptorelin plus $6500 \mathrm{IU}$ of recombinant hCG for trigger.

Results: The dual-trigger group had significantly higher oocyte fertilization rate (73.1\% vs. 58.6\%), clinical pregnancy rate (33. $0 \%$ vs. $20.7 \%$ ) and live birth rate (26.9\% vs. $14.5 \%$ ) when compared to the hCG trigger group. In addition, the abortion rate $(17.4 \%$ vs. $37.0 \%)$ and embryo transfer cancellation rate (6.1\% vs. $15.4 \%)$ were both significantly lower in the dual trigger group. The primary outcome measure was the live birth rate per oocyte retrieval cycle. Secondary outcome measures were embryo transfer cancellation rate, clinical pregnancy rate, implantation rate, chemical pregnancy rate and abortion rate per oocyte retrieval cycle.
\end{abstract}

Conclusions: Dual triggering the final oocyte maturation with GnRH-a and standard dose of $\mathrm{hCG}$ can significantly improve the live birth rate, clinical pregnancy rate, and fertilization rate in women with diminished ovarian reserve undergoing $\mathrm{GnRH}$ antagonist down-regulated IVF-ICSI cycles.

Keywords: Dual trigger, Diminished ovarian reserve, IVF, GnRH agonist

\footnotetext{
* Correspondence: hwuba4416@gmail.com

${ }^{+}$Ming-Huei Lin and Frank Shao-Ying Wu contributed equally to this work.

'Department of Obstetrics and Gynecology, Mackay Memorial Hospital,

Taipei, Taiwan

${ }^{2}$ Department of Medical Research, Mackay Memorial Hospital, Taipei, Taiwan

Full list of author information is available at the end of the article
}

(c) The Author(s). 2019 Open Access This article is distributed under the terms of the Creative Commons Attribution 4.0 International License (http://creativecommons.org/licenses/by/4.0/), which permits unrestricted use, distribution, and reproduction in any medium, provided you give appropriate credit to the original author(s) and the source, provide a link to the Creative Commons license, and indicate if changes were made. The Creative Commons Public Domain Dedication waiver (http://creativecommons.org/publicdomain/zero/1.0/) applies to the data made available in this article, unless otherwise stated. 


\section{Background}

The management of the "diminished ovarian reserve" (DOR) or poor responder to ovarian stimulation remains one of the most challenging aspects in the field of fertility treatment. According to the data published by Society for Assisted Reproductive Technologies, the number of women aged 40 years or older seeking fertility treatment had increased by more than 80\% between 1999 and 2008 [1]. Therefore, it came as no surprise that the population of poor responders has grown exponentially over the years. It has been estimated of all couples treated in IVF units, $10-24 \%$ of them were poor responders [2].

In attempts to optimize IVF cycle outcomes for poor responders, modifying the steps of conventional ovarian stimulation protocols have been proposed, such as different luteal phase pretreatments, ovarian stimulation protocols, as well as addition of various supplements. Though most of the modifications had limited success, an optimal protocol for poor responders has remained elusive [3].

Nevertheless, one of the most critical steps during IVF treatment has remained mostly untouched, which is the use of human chorionic gonadotropin (hCG) for triggering final oocyte maturation before retrieval. The discovery of hCG was made by Ascheim and Zondek nearly ninety years ago [4]. From the blood and urine of pregnant women, they extracted an injectable gonad-stimulating substance that could induce follicular maturation and ovarian stromal luteinization in immature mice. Until now, administering $5000 \mathrm{IU}$ to $10,000 \mathrm{IU}$ of hCG 34-36 h prior to oocyte retrieval still remained as the standard protocol for the induction of final oocyte maturation in IVF cycles worldwide.

As an effort to eliminate the incidence of ovarian hyperstimulation (OHSS), substitution of hCG with gonadotropinreleasing hormone agonist ( $\mathrm{GnRH}-\mathrm{a})$ for trigger was proposed for IVF high responders [5, 6]. Triggering with GnRH-a indeed have minimized the risk of OHSS, but the pregnancy rate was found to be adversely affected due to the impaired luteal function $[5,6]$. In order to rescue the luteal phase, strategies such as aggressive post-retrieval progesterone and estrogen supplementation [7], or adding a reduced dose of hCG either at oocyte retrieval [8] or intermittently during luteal phase [9] were implemented. Then the concept of "dual trigger" with GnRH-a plus a reduced dose of hCG emerged as another mean to correct the dysfunctional luteal phase after $\mathrm{GnRH}$-a triggering. In a study consisted of high responders, Shapiro et al. first demonstrated that acceptable rates of fertilization, implantation, clinical pregnancy, ongoing pregnancy, and early pregnancy loss could still be obtained using a dual trigger regimen consisted of $4 \mathrm{mg}$ leuprolide acetate and 1000 to $2500 \mathrm{IU}$ of hCG [10]. In two follow-up studies on high responders, the group that used dual trigger consistently showed superior ongoing pregnancy rate [11] and live birth rate [12] when compared to the group that used GnRH-a trigger alone. Most importantly, the use of dual trigger was not associated with the increased incidence of OHSS [10-12].

In two similar studies consisted of normal responders undergoing IVF, significant improvement in total number of retrieved oocytes [13], number of mature (MII) oocytes [13], rates of embryo implantation [13], clinical pregnancy [13, 14], ongoing pregnancy [14] and live birth [13] were also observed when dual trigger was used instead of hCG trigger. These findings further indicated that in addition to reducing the incidence of OHSS, the GnRH-a component in the dual trigger could also have important roles in oocytes maturation and embryo implantation.

In light of the promising results demonstrated for the high and normal responder groups, the present study is set to investigate whether dual trigger with standard dose hCG and GnRH-a can also have a role in improving the IVF cycle outcomes among patients of diminished ovarian reserve.

\section{Methods \\ Study design}

This is a retrospective cohort analysis consisted of $\mathrm{GnRH}$ antagonist IVF cycles from October, 2013 through December, 2016 at the Infertility Division of Mackay Memorial Hospital in Taipei City, Taiwan. The study protocol was approved by the institutional review board of Mackay Memorial Hospital.

\section{Study participants}

In the present study, diminished ovarian reserve was defined as the presence of both serum anti-Müllerian hormone level $(\leq 1.1 \mathrm{ng} / \mathrm{mL})$ and low antral follicle count $(\leq$ 5 ) at the time of initiation of ovarian stimulation. Exclusion criteria were co-existing endocrine disorders (diabetes mellitus, hyperprolactinemia, thyroid dysfunction, congenital adrenal hyperplasia, Cushing syndrome), untreated hydrosalpinx, and uterine anomaly confirmed either by hysterosalpingography or hysteroscopy. After applying the exclusion criteria, a total of 427 IVF cycles with fresh embryo transfer (ET) were included for final analysis (hCG trigger/control group: $n=130$; dual trigger/study group: $n=297$ ).

\section{Ovarian stimulation protocols}

After a baseline pelvic ultrasound, controlled ovarian stimulation was commenced on the third day of the menstrual cycle for three consecutive days with a fixed starting dose of r-FSH (Gonal-F; Merck Serono Biopharma) of 450 IU for all subjects. The starting dose was chosen based on observations from previous studies $[15,16]$ that demonstrated no added benefit to daily gonadotropin dosing above 450 IU. The daily r-FSH dose was then adjusted according to ovarian response. When at least one follicle reached $14 \mathrm{~mm}$ in diameter, $\mathrm{GnRH}$ antagonist (Cetrotide; 
Merck Serono Biopharma) was added to the stimulation protocol. When at least two leading follicles reached 18 $\mathrm{mm}$ in diameter, induction of final oocyte maturation was triggered either by $6500 \mathrm{IU}$ of recombinant hCG (Ovidrel; Merck Serono Biopharma), or by a combination of 6500 IU of recombinant hCG and $0.2 \mathrm{mg}$ of triptorelin (Decapeptyl; Ferring Pharmaceuticals). The choice of triggering method was based on the attending physicians' discretion.

Oocyte retrievals were performed under transvaginal ultrasound guidance, 35 to $36 \mathrm{~h}$ post triggering. Standard insemination procedures were performed for all cases, with exceptions in cases with male factor, for which intracytoplasmic sperm injections (ICSI) were performed instead. All embryo transfers were performed on day-3 after oocyte retrieval.

\section{Luteal phase support and confirmation of pregnancy}

Luteal phase supplement with daily vaginal progesterone gel (Crinone 8\%; Merck Serono Biopharma) was initiated on the day of oocyte retrieval. Serum beta-hCG level was measured 14 days post oocyte retrieval, and a value above $5 \mathrm{IU} / \mathrm{mL}$ was considered as positive pregnancy. The luteal phase support was then continued until the 10th weeks of gestation for all positive pregnancies.

\section{Outcome measures}

The primary outcome measure was the live birth rate per oocyte retrieval cycle. Secondary measures were embryo transfer cancellation rate, clinical pregnancy rate, implantation rate, chemical pregnancy rate, and abortion rate per oocyte retrieval cycle. Embryo transfer cancellation was defined as discontinuation of embryo transfer due to fertilization failure or embryonic cleavage arrest. Live birth was defined as delivery of a viable fetus of $>23$ weeks of gestation. Clinical pregnancy was defined as the visualization of the fetal heart beat by ultrasound between the 5th to 6th weeks of gestation. The implantation rate was calculated as the total number of observed gestational sacs divided by the total number of transferred embryos. Biochemical pregnancy was defined as a transient positive serum beta-hCG level without subsequent development of visible gestational sac. Abortion was defined as blighted ovum or fetal demise before 20th weeks of gestation.

\section{Statistical analysis}

The statistical analysis was performed using MedCalc 10.2 (MedCalc Software, Mariakerke, Belgium). Continuous variables were presented as mean with standard deviations (SD), and the between-group differences were analyzed by independent Student's $\mathrm{t}$-test. Categorical variables were presented as raw frequencies with corresponding percentages, and the between-group differences were analyzed either by Chi-square test with
Yates correction if indicated, or by Fisher exact test. A $P$-value $<0.05$ was considered as statistically significant.

\section{Results}

The baseline characteristics for the control and the study group are summarized in Table 1. The was no significant differences in the patient age, serum AMH level, and etiologies of infertility. The response to ovarian stimulation and cycle outcomes are presented in Table 2. There was no significant difference in the total r-FSH dose, duration of stimulation, endometrial thickness, and hCG day serum hormone profiles between the control and the study group. The mean number of total retrieved oocytes and mature metaphase II (MII) oocytes were also similar between the two groups (Table 2). In terms of cycle outcome, the dual-trigger group demonstrated a significantly higher fertilization rate $(73.1 \%$ vs. $58.6 \% ; P=.015)$, clinical pregnancy rate $(33.0 \%$ vs. $20.7 \%, P=.035)$ and live birth rate $(26.9 \%$ vs. $14.5 \%, P=.014)$ compared to the hCG trigger group (Table 3$)$. The cycle cancellation rate $(6.1 \%$ vs. $15.4 \%$, $P=.003)$ and abortion rate were significantly lower in the dual trigger group $(17.4 \%$ vs.37.0\%, $P=.037)$. There was no incidence of OHSS in either group.

\section{Discussion}

The present study offered a novel strategy for improving the outcomes in patients with diminished ovarian reserve undergoing GnRH antagonist down-regulated IVF-ICSI cycles. By triggering the final oocyte maturation with a combination of standard dose hCG and GnRH-a, the study group demonstrated a significantly higher oocyte fertilization rate, clinical pregnancy rate and live birth rate, while incurring a lower embryo transfer cancellation rate and abortion rate when compared to the control group. The observed benefits of using the dual-trigger concurred with previous reports [17-19], although the data on the live birth rate was not provided in those studies. To the best of our knowledge, this is the first ever study that demonstrated the positive effects of dual triggering in diminished ovarian reserve population in terms of improved live birth rate.

Ever since its inauguration in 2011, the Bologna Criteria has been one of most adopted guidelines for defining poor responders [20]. Nevertheless, the above classification is not without limitation. For example, in a previous report that showed that dual trigger significantly increased the number of oocytes retrieved and the oocyte maturation rate in poor responders, the authors only recruited the subjects that fulfilled the Bologna criteria [18]. By applying such arbitrary outline, other "potential" poor responders who were under 40 years of age, as well as ones without previous IVF stimulation cycles would had been excluded. In the present study, we used diminished ovarian reserve tests $(\mathrm{AMH}<1.1 \mathrm{ng} / \mathrm{ml}$ and $\mathrm{AFC} \leq 5)$ as our primary 
Table 1 Comparison between hCG and dual-trigger protocols: Patient demographics

\begin{tabular}{llll}
\hline Patients 'baseline profiles & Control group (hCG) & Study group (hCG + triptorelin) & $P$ value \\
\hline Total number of egg retrieval cycles & 130 & 297 & - \\
Age $(\mathrm{y})$ & $38.26 \pm 3.27$ & $37.85 \pm 3.73$ & NS \\
AMH $(\mathrm{ng} / \mathrm{ml})$ & $0.81 \pm 0.26$ & $0.84 \pm 0.23$ & $\mathrm{NS}$ \\
Etiology of infertility (\%) & & 28.8 & NS \\
Male Factor & 37.1 & 29.6 & NS \\
Tubal Factor & 30.7 & 50.4 & NS \\
Ovulation Dysfunction & 51.3 & 41.2 & NS \\
Endometriosis & 41.6 & 4.4 & NS \\
Uterine factor & 2.2 & 4.5 & NS \\
Unexplained & 10.5 & & \\
\hline
\end{tabular}

Note: Values are expressed as mean \pm standard deviation or percentage. AMH anti-Müllerian hormone, hCG human chorionic gonadotropin, NS non-statistically significant

inclusion criteria. Thus, more patients with low oocytes yield could be included in our analysis, which enable the results derived from the study to be applicable to broader populations and clinical settings.

One of the advantages of triggering with GnRH-a is the simultaneous induction of a mid-cycle FSH surge that is similar to the hormonal events in a natural ovulatory cycle. Animal studies have confirmed the importance of FSH in up regulating of luteinizing hormone (LH) receptor sites formations in granulosa cells [21, 22]. The expression of LH receptors is essential for preparing the maturing follicle for the pre-ovulatory LH surge and the luteinzation of granulosa cells. FSH also has a key role in promoting the resumption of oocyte meiosis $[23,24]$ and the expansion of cumulus cells $[25,26]$, all of which are critical steps in the oocyte maturation process. Therefore, one of the proposed benefits of $\mathrm{GnRH}$-a triggering is the increased rate of mature oocytes retrieved. From the studies focusing on high responders [5, 8] normal responders [13], and poor responder (18), the GnRH-a trigger group had consistently resulted in higher proportion of metaphase II (MII) oocytes at the time of retrieval compared to the hCG group. In a related study by Griffin et al., patients with a history of $>25 \%$ of immature oocytes at previous retrieval had marked improvement of oocyte maturity when dual-triggered with standard dose hCG and GnRH-a in subsequent IVF cycles [27]. Though similar effects of improved oocyte maturation was not observed in the current study, this was not unexpected since low oocyte yield was anticipated for this specific study population, and a statistically significant difference would have been difficult to observe with the present sample size. Nevertheless, the trend toward improved implantation and reduced chemical pregnancy rates, as well as significantly higher rates of oocyte fertilization, clinical pregnancy and live birth showed in the present study could also be viewed as an enhancement of oocyte competence from dual triggering. In a related study, the authors simulated an artificial mid-cycle FSH surge by adding a single bolus of FSH (450 IU) to the hCG as trigger [28]. Compared to the control group triggered by hCG and placebo, the study group triggered by FSH and hCG had significantly improved oocyte competence, as demonstrated by greater oocyte recovery and fertilization rate. In another report, a case of repetitive immature oocytes and empty follicle syndrome was also successfully treated with dual triggering, resulting in a singleton live birth at term [29].

Furthermore, in a pilot study by Haas et al., the differential messenger RNA (mRNA) expression of reproduction-related genes in the oocyte granulosa cells (GCs) of patients triggered with hCG were compared to the same cohorts triggered with GnRH-a plus hCG (dual

Table 2 Comparison between hCG and dual-trigger protocols: Outcomes of ovarian stimulations

\begin{tabular}{llll}
\hline & Control group $(\mathrm{hCG})$ & Study group (hCG + triptorelin) & $P$ value \\
\hline$E_{2}$ on trigger day $(\mathrm{pg} / \mathrm{ml})$ & $775.29 \pm 500.1$ & $628.16 \pm 503.4$ & NS \\
No. of oocytes Retrieved & $3.40 \pm 1.36$ & $3.27 \pm 1.53$ & NS \\
No. of Mll oocytes Retrieved & $2.85 \pm 1.33$ & $2.75 \pm 1.42$ & NS \\
Fertilization rate $(\%)$ & $58.63 \pm 36.23$ & $73.10 \pm 71.80$ & 0.015 \\
No. of embryo transferred & $1.67 \pm 1.10$ & $2.06 \pm 1.11$ & NS \\
\hline
\end{tabular}

Note: Values are expressed as mean \pm standard deviation or percentage. $E_{2}$ estradiol, MII metaphase II, hCG human chorionic gonadotropin, NS non-statistically significant 
Table 3 Comparison between hCG and dual-trigger protocols: Outcomes of IVF-ICSI cycles

\begin{tabular}{llll}
\hline & Control group (hCG) & Study group (hCG + triptorelin) & $P$ value \\
\hline Cancellation of embryo transfer (\%) & $15.4(20 / 130)$ & $6.1(18 / 297)$ & 0.003 \\
Implantation Rate (\%) & $10.58 \pm 2.45$ & $15.02 \pm 1.74$ & NS \\
Biochemical pregnancy rate per cycle (\%) & $4.6(6 / 130)$ & $2.0(6 / 297)$ & $\mathrm{NS}$ \\
Clinical pregnancy rate per cycle (\%) & $20.7(27 / 130)$ & $33.0(92 / 297)$ & 0.035 \\
Live birth rate per cycle (\%) & $13.1(17 / 130)$ & $27.2(76 / 297)$ & 0.014 \\
Abortion rate (\%) & $37.0(10 / 27)$ & $4.4(16 / 92)$ & 0.037 \\
Twin pregnancy rate (\%) & $1.5(2 / 130)$ & NS \\
\hline
\end{tabular}

Note: Values are expressed as mean \pm standard deviation or percentage. $h$ CG human chorionic gonadotropin, NS non-statistically significant

trigger) in the subsequent IVF cycles [30]. The authors found that higher levels of amphiregulin and epiregulin were expressed in the GCs after dual triggering. Amphiregulin and epiregulin are ligands of the epidermal growth factor (EGF) receptors, and both have been indicated to participate important roles in cumulus expansion [31,32], oocytes maturation [32], and meiosis resumption [31]. Since both amphiregulin and epiregulin expressions are up-regulated directly in the presence of FSH and $\mathrm{LH}$, the surges of these two hormone induced by the GnRH-a trigger may be one of the mechanisms responsible for the improved fertilization, clinical pregnancy rate and live birth rate observed in the present study.

Interestingly, Haas et al. had also reported a modified "double trigger" protocol aimed for patients with low proportion of mature oocytes per follicle in the preceding IVF cycles $[19,33]$. Specifically, the GnRH-a and hCG was administered $6 \mathrm{~h}$ apart, at 40 and $34 \mathrm{~h}$ prior to oocyte retrieval, respectively. When compared to previous cycles triggered with hCG alone, the subjects in both studies showed significant higher number of retrieved oocyte, 2PN embryos, available embryo for transfer, and ongoing pregnancy rate when modified trigger was used. Though the two studies contained very small sample sizes $(n=8$; $n=12$ ), their results also indicated improvement in oocyte and embryo quality when $\mathrm{GnRH}-\mathrm{a}$ was included as part of trigger regimen along with hCG. In addition to further affirm the observations made from the present study, these results also showed that the GnRH-a component can either be administered simultaneously with hCG or preceding it without losing its positive effects. A summary of all the reported $\mathrm{GnRH}-\mathrm{a}$ and hCG trigger combinations are provided in the comprehensive review by Orvieto [34]. More importantly, the article is one of the first to provide an individualized patient-based approach in choosing the most suitable trigger regimen for different subgroups of infertility patients, which not only can optimize the treatment outcomes but also minimize the risks of OHSS.

In addition, another proposed advantage with dual trigger is potential enhancement of endometrial receptivity by the $\mathrm{GnRH}-\mathrm{a}$ component. Significant elevation of both isoforms of human $\mathrm{GnRH}$ mRNA expression have been detected in the secretory phase of the human menstrual cycle [35-37], indicating the possible role of these hormones in regulation of endometrial receptivity [35, 38]. Specifically, in vitro studies with human extra-villous cytotrophoblasts and decidual stroma cells have demonstrated the ability of GnRH to activate urokinase type plasminogen activator, a key component in decidualization and trophoblast invasion [39, 40]. Therefore, inclusion of $\mathrm{GnRH}-\mathrm{a}$ as part of luteal support regimen has been explored as a mean to improve the implantation rate. In a related meta-analysis [41], the authors concluded that a single-dose of GnRH-a in the luteal phase could significantly enhance the implantation rates in either $\mathrm{GnRH}-\mathrm{a}$ or GnRH-antagonist down-regulated ICSI cycles. Interestingly, improvement in clinical and ongoing pregnancy rates with luteal $\mathrm{GnRH}$-a were only observed in $\mathrm{GnRH}$ antagonist downregulated cycles. Though the dual trigger in the present study was administered in the pre-ovulation period, we believe with the strong affinity of $\mathrm{GnRH}-\mathrm{a}$ to its receptors as well as its longer half-lives, GnRH-a could still exert its positive effects during embryo implantation period. In the previous studies consisted of normal responders, the dual-riggered group had consistently demonstrated higher implantation rates when compared to conventional hCG triggered group $[13,14]$. Schacter et al. postulated the positive effect of pre-ovulatory GnRH-a administration on embryo implantation could be attributed to displacement of the GnRH-ant from the endometrial GnRH receptors by the GnRH-a, thus enabling proper post-receptor actions for implantation [14].

With evolving clinical acceptance and understanding, triggering with $\mathrm{GnRH}-\mathrm{a}$ has since become an integral part of modern IVF practices, especially in high responders, oocytes donors, as well as oncology patients seeking fertility preservation. Nevertheless, other than the known hypothalamic hypogonadism patients, there remains a subsets infertile patients who are not suitable candidates for triggering with GnRH-a alone. In a retrospective analysis by Meyers et al., the overall incidence of suboptimal response to GnRH-a trigger was reported to be $5.2 \%$, with long term oral contraceptive use and low serum LH level $(\leq 0.5 \mathrm{mIU} / \mathrm{mL})$ on the day of triggering identified as the most relevant risk factors [42]. Specifically, suboptimal response was defined as an inadequate hormone surge with low serum LH level $(<15 \mathrm{mIU})$ 
drawn 8-12 h after ovulatory trigger. All subjects were young age, presumed normal responders triggered by either $\mathrm{GnRH}$-a alone or by dual trigger (low-dose hCG (1000-3300 IU) plus GnRH-a). In contrast to the previous reports by the Humaidan group (8), the rationale of adding a low dose hCG was intended as an alternative trigger rather than provide luteal support. Since the rate of suboptimal response to the GnRH-a trigger reported was $5.2 \%$ [42], it seemed the hCG component in the dual trigger can at least partially compensate the inadequate response to GnRH-a induced hormone surge.

From the above data, we proposed the GnRH-a component in the dual trigger has two distinct yet related functions. In an elegant study by Fauser et al., the authors demonstrated that the GnRH-a trigger would induced a rapid LH and FSH surge at $4 \mathrm{~h}$ and $8 \mathrm{~h}$ post injection, respectively [43]. Therefore, the area under curve (AUC) of serum FSH and LH concentrations within the first $24 \mathrm{~h}$ for oocytes maturation would be much higher from the GnRH-a trigger compared to the hCG trigger. As a result, dual trigger may impose an earlier and more potent oocyte maturation effects than hCG trigger alone within first $24 \mathrm{~h}$. Since the LH surge induced by GnRH-a occurs more rapidly than the hCG triggering peak $4 \mathrm{~h}$ vs. $12-24$ h) [43], the hCG component of the dual trigger could serve as an second / rescue trigger in the event of poor response to $\mathrm{GnRH}-\mathrm{a}$ as described in the report by Myers et al. [42]. Secondly, the bolus of hCG could also correct the defective luteal phase resulted from the GnRH-a trigger, as described in the reports 8). Though the timing and dosing of hCG administration in the present study were different from the reports by Humaidan group [8], the rationale of utilizing hCG as part of enhanced luteal support was essentially the same.

In conclusion, the results from the present study demonstrated that in GnRH antagonist down-regulated IVF-ICSI cycles, dual triggering the final oocyte maturation with GnRH-a and standard dose of hCG could significantly improve the rate of fertilization, clinical pregnancy as well as live birth in women of diminished ovarian reserve. Furthermore, the benefit of lowered cycle cancellation rate would also enable greater percentage of patients with diminished ovarian reserve to reach the final stage of their IVF treatment, thereby enhancing their chance of achieving a successful pregnancy as well as alleviating the psychological stress. The limitation of the present study is its retrospective design. Future prospective randomized controlled studies, though difficult, are still required to affirm the beneficial effects of dual triggering in diminished ovarian reserve. Nevertheless, the data from the present study concurred with other studies of dual triggering, which further confirmed the proof-of-concept that calls for a possible paradigm shift in ovulation-triggering agent for GnRH-antagonist cycles.

\section{Abbreviations}

FSH: Follicular stimulating hormone; GnRH: Gonadotropin releasing hormone; hCG: Human chorionic gonadotropin; ICSI: Intracytoplasmic sperm injection; IVF: In vitro fertilization; OHSS: Ovarian hyperstimulation syndrome

\section{Acknowledgements}

Not applicable.

Funding

Not applicable.

Availability of data and materials

All data are available from the corresponding author upon request.

\section{Authors' contributions}

$\mathrm{M}-\mathrm{HL}$ and FS-YW contributed equally to the study. M-HL and Y-MH Data collection, study design, clinical treatments and follow-ups. FS-YW Data integration and interpretation, manuscript preparation. RK-KL and S-HL Supervision of data quality control and laboratory procedures. R-SL Statistical analysis of data. All authors read and approved the final manuscript.

\section{Ethics approval and consent to participate}

The study was approved by the Institution Review Board of Clinical Trial at MacKay Memorial Hospital. (IRB Number: 18MMHIS074e).

Consent for publication

All authors agree in publishing the data and results contained in this article.

\section{Competing interests}

The authors declare that they have no competing interests.

\section{Publisher's Note}

Springer Nature remains neutral with regard to jurisdictional claims in published maps and institutional affiliations.

\section{Author details}

${ }^{1}$ Department of Obstetrics and Gynecology, Mackay Memorial Hospital, Taipei, Taiwan. ${ }^{2}$ Department of Medical Research, Mackay Memorial Hospital, Taipei, Taiwan. ${ }^{3}$ Mackay Junior College of Medicine, Nursing, and Management, Taipei, Taiwan. ${ }^{4}$ Mackay Medical College, New Taipei City, Taiwan. ${ }^{5}$ HMED Fertility Clinic, Taipei, Taiwan. ${ }^{6}$ Taipei City Hospital, Heping-Fuyou Branch, Taipei, Taiwan. ${ }^{7}$ Department of Obstetrics and Gynecology, Taipei Medical University, Taipei, Taiwan.

Received: 9 November 2018 Accepted: 27 December 2018

Published online: 04 January 2019

\section{References}

1. Patrizio P, Vaiarelli A, Levi Setti PE, Tobler KJ, Shoham G, Leong M, Shoham Z. How to define, diagnose and treat poor responders? Responses from a worldwide survey of IVF clinics. Reprod BioMed Online. 2015;30:581-92.

2. Keay SD, Liversedge NH, Mathur RS, Jenkins JM. Assisted conception following poor ovarian response to gonadotrophin stimulation. Br J Obstet Gynaecol. 1997;104:521-7.

3. Ubaldi F, Vaiarelli A, D'Anna R, Rienzi L. Management of poor responders in IVF: is there anything new? Biomed Res Int. 2014;2014:352098.

4. Ascheim S, Zondec B. Hypophysenvorderlappen-hormone und ovarial hormone imHarn von schwangeren. Klinwochenscher. 1927;6:13-21.

5. Humaidan $P$, Bredkjaer HE, Bungum L, Bungum M, Grondahl ML, Westergaard L, et al. GnRH agonist (buserelin) or hCG for ovulation induction in $\mathrm{GnRH}$ antagonist IVF/ICSI cycles: a prospective randomized study. Hum Reprod. 2005;20:1213.

6. Kolibianakis EM, Schultze-Mosgau A, Schroer A, van Steirteghem A, Devroey $P$, Diedrich $K$, Griesinger $G$. A lower ongoing pregnancy rate can be expected when GnRH agonist is used for triggering final oocyte maturation instead of hCG in patients undergoing IVF with GnRH antagonists. Hum Reprod. 2005;20:2887-92.

7. Engmann L, DiLuigi A, Schmidt D, Nulsen J, Maier D, Benadiva C. The use of gonadotropin-releasing hormone $(\mathrm{GnRH})$ agonist to induce oocyte maturation after cotreatment with $\mathrm{GnRH}$ antagonist in high-risk patients 
undergoing in vitro fertilization syndrome: a prospective randomized controlled study. Fertil Steril. 2008:89:84-91.

8. Humaidan P, EjdrupBredkjaer H, Westergaard LG, Yding Andersen C. 1,500 IU human chorionic gonadotropin administered at oocyte retrieval rescues the luteal phase when gonadotropin-releasing hormone agonist is used for ovulation induction: a prospective, randomized, controlled study. Fertil Steril. 2010;93:847-54.

9. Castillo JC, Dolz M, Bienvenido E, Abad L, Casan EM, Bonilla-Musoles F. Cycles triggered with GnRH agonist: exploring low-dose hCG for luteal support. Reprod BioMed Online. 2010;20:175-81.

10. Shapiro BS, Daneshmand ST, Garner FC, Aguirre M, Thomas S. Gonadotropin-releasing hormone agonist combined with a reduced dose of human chorionic gonadotropin for final oocyte maturation in fresh autologous cycles of in vitro fertilization. Fertil Steril. 2008;90:231-3.

11. Shapiro BS, Daneshmand ST, Garner FC, Aguirre M, Hudson C. Comparison of "triggers" using leuprolide acetate alone or in combination with lowdose human chorionic gonadotropin. Fertil Steril. 2011;95:2715-7.

12. Griffin D, Benadiva C, Kummer N, Budinetz T, Nulsen J, Engmann L. Dual trigger of oocyte maturation with gonadotropin-releasing hormone agonist and low-dose human chorionic gonadotropin to optimize live birth rates in high responders. Fertil Steril. 2012;97: 1316-20.

13. Lin $M H$, Wu FS, Lee RK, Li SH, Lin SY, Hwu YM. Dual trigger with combination of gonadotropin-releasing hormone agonist and human chorionic gonadotropin significantly improves the live-birth rate for normal responders in GnRH-antagonist cycles. Fertil Steril. 2013;100:1296-302.

14. Schachter M, Friedler S, Ron-El R, Zimmerman AL, Strassburger D, Bern O, et al. Can pregnancy rate be improved in gonadotropin-releasing hormone $(\mathrm{GnRH})$ antagonist cycles by administering $\mathrm{GnRH}$ agonist before oocyte retrieval? A prospective, randomized study. Fertil Steril. 2008:90:1087-93.

15. Land JA, Yarmolinskaya MI, Dumoulin JC, Evers JL. High-dose human menopausal gonadotropin stimulation in poor responders does not improve in vitro fertilization outcome. Fertil Steril. 1996;65:961-5.

16. Manzi DL, Thornton KL, Scott LB, Nulsen JC. The value of increasing the dose of human menopausal gonadotropins in women who initially demonstrate a poor response. Fertil Steril. 1994:62:251-6.

17. Orvieto R. A simplified universal approach to $\mathrm{COH}$ protocol for IVF: ultrashort flare GnRH-agonist/GnRH- antagonist protocol with tailored mode and timing of final follicular maturation. J Ovarian Res. 2015;8:69.

18. Zhang J, Wang Y, Mao X, Chen Q, Hong Q, Cai R, et al. Dual trigger of final oocyte maturation in poor ovarian responders undergoing IVF/ICSI cycles. Reprod BioMed Online. 2017;35:701-7.

19. Zilberberg E, Haas J, Dar S, Kedem A, Machtinger R, Orvieto R. Coadministration of GnRH-agonist and hCG, for final oocyte maturation (double trigger), in patients with low proportion of mature oocytes. Gynecol Endocrinol. 2015;31:145-7.

20. Ferraretti AP, La Marca A, Fauser BC, Tarlatzis B, Nargund G, Gianaroli L. ESHRE working group on poor ovarian response definition. ESHRE consensus on the definition of "poor response" to ovarian stimulation for in vitro fertilization: the Bologna criteria. Hum Reprod. 2011;26:1616-24.

21. Richards JS, Ireland JJ, Rao MC, Bernath GA, Midgley AR Jr, Reichert LE Jr. Ovarian follicular development in the rat: hormone receptor regulation by estradiol, follicle stimulating hormone and luteinizing hormone. Endocrinology. 1976;99:1562-70.

22. Zeleznik AJ, Midgley AR, Reichert LE Jr. Granulosa cell maturation in the rat: increased binding of human chorionic gonadotropin following treatment with follicle stimulating hormone in vitro. Endocrinology. 1974;95:818-25.

23. Yding Andersen C, Leonardsen L, Ulloa-Aguirre A, Barrios-De-Tomasi J, Moore L, Byskov AG. FSH-induced resumption of meiosis in mouse oocytes: effect of different isoforms. Mol Hum Reprod. 1999;5:726-31.

24. Zelinski-Wooten MB, Hutchison JS, Hess DL, Wolf DP, Stouffer RL. Follicle stimulating hormone alone supports follicle growth and oocyte development in gonadotrophin-releasing hormone antagonisttreated monkeys. Hum Reprod. 1995:10:1658-66.

25. Eppig JJ. FSH stimulates hyaluronic acid synthesis by oocyte-cumulus cell complexes from mouse preovulatory follicles. Nature. 1979;281:483-4.

26. Stickland S, Beers WH. Studies on the role of plasminogen activator in ovulation. In vitro response of granulosa cells to gonadotropins, cyclic nucleotides, and prostaglandins. J Biol Chem. 1976;251:5694-702.

27. Griffin D, Feinn R, Engmann L, Nulsen J, Budinetz T, Benadiva C. Dual trigger with gonadotropin-releasing hormone agonist and standard dose human chorionic gonadotropin to improve oocyte maturity rates. Fertil Steril. 2014:102:405-9.

28. Lamb JD, Shen S, McCulloch C, Jalalian L, Cedars MI, Rosen MP. Folliclestimulating hormone administered at the time of human chorionic gonadotropin trigger improves oocyte developmental competence in in vitro fertilization cycles: a randomized, double-blind, placebo-controlled trial. Fertil Steril. 2011;95:1655-60

29. Castillo JC, Moreno J, Dolz M, Bonilla-Musoles F. Successful pregnancy following dual triggering concept (rhCG + GnRH agonist) in a patient showing repetitive Inmature oocytes and empty follicle syndrome: case report. J Med Cases. 2013:4:221-6.

30. Haas J, Ophir L, Barzilay E, Machtinger R, Yung Y, Orvieto R, Hourvitz A. Standard human chorionic gonadotropin versus double trigger for final oocyte maturation results in different granulosa cells gene expressions: a pilot study. Fertil Steril. 2016:106:653-9.

31. Caixeta ES, Machado MF, Ripamonte P, Price C, Buratini J. Effects of FSH on the expression of receptors for oocyte-secreted factors and members of the EGF-like family during in vitro maturation in cattle. Reprod Fertil Dev. 2013;25:890-9.

32. Park JY, Su YQ, Ariga M, Law E, Jin SL, Conti M. EGF-like growth factors as mediators of LH action in the ovulatory follicle. Science. 2004;303:682-4.

33. Haas J, Zilberberg E, Dar S, Kedem A, Machtinger R, Orvieto R. Coadministration of GnRH-agonist and hCG for final oocyte maturation (double trigger) in patients with low number of oocytes retrieved per number of preovulatory follicles--a preliminary report. J Ovarian Res. 2014;2(7):77.

34. Orvieto R. Triggering final follicular maturation-hCG, GnRH-agonist or both, when and to whom? J Ovarian Res. 2015:8:60

35. Cheon KW, Lee HS, Parhar IS, Kang IS. Expression of the second isoform of gonadotrophin-releasing hormone (GnRH-II) in human endometrium throughout the menstrual cycle. Mol Hum Reprod. 2001;7:447-52.

36. Dong KW, Marcelin K, Hsu MI, Chiang CM, Hoffman G, Roberts JL. Expression of gonadotropin-releasing hormone $(\mathrm{GnRH})$ gene in human uterine endometrial tissue. Mol Hum Reprod. 1998;4:893-8.

37. Raga F, Casan EM, Kruessel JS, Wen Y, Huang HY, Nezhat C, Polan ML. Quantitative gonadotropin-releasing hormone gene expression and immune histochemical localization in human endometrium throughout the menstrual cycle. Biol Reprod. 1998;59:661-9.

38. Raga F, Casan EM, Wen Y, Huang HY, Bonilla-Musoles F, Polan ML Independent regulation of matrix metalloproteinase-9, tissue inhibitor of metalloproteinase-1 (TIMP-1), and TIMP-3 in human endometrial stromal cells by gonadotropin-releasing hormone: implications in early human implantation. J Clin Endocrinol Metab. 1999;84:636-42.

39. Chou CS, MacCalman CD, Leung PC. Differential effects of gonadotropinreleasing hormone I and II on the urokinase-type plasminogen activator/ plasminogen activator inhibitor system in human decidual stromal cells in vitro. J Clin Endocrinol Metab. 2003;88:3806-15.

40. Paria BC, Reese J, Das SK, Dey SK. Deciphering the cross-talk of implantation: advances and challenges. Science. 2002;296:2185-8.

41. Oliveira JB, Baruffi R, Petersen CG, Mauri AL, Cavagna M, Franco JG Jr. Administration of single-dose GnRH agonist in the luteal phase in ICSI cycles:a meta-analysis. Reprod Biol Endocrinol. 2010;8:107.

42. Meyer L, Murphy LA, Gumer A, Reichman DE, Rosenwaks Z, Cholst IN. Risk factors for a suboptimal response to gonadotropin-releasing hormone agonist trigger during in vitro fertilization cycles. Fertil Steril. 2015;104:637-42.

43. Fauser BC, de Jong D, Olivennes F, Wramsby H, Tay C, Itskovitz-Eldor J, et al. Endocrine profiles after triggering of final oocyte maturation with $\mathrm{GnRH}$ agonist after cotreatment with the GnRH antagonist ganirelix during ovarian hyperstimulation for in vitro fertilization. J Clin Endocrinol Metab. 2002;87:709-15

\section{Ready to submit your research? Choose BMC and benefit from:}

- fast, convenient online submission

- thorough peer review by experienced researchers in your field

- rapid publication on acceptance

- support for research data, including large and complex data types

- gold Open Access which fosters wider collaboration and increased citations

- maximum visibility for your research: over $100 \mathrm{M}$ website views per year

At BMC, research is always in progress.

Learn more biomedcentral.com/submissions 